as control. HUVECs were infected with Ad-XBP1 or Ad-GFP for $48 \mathrm{~h}$, then stimulated in the presence or absence of TNF- $\alpha$ (10 ng/ $\mathrm{ml}$ ) for $24 \mathrm{~h}$. Intracellular superoxide anions generated by HUVECS were measured by Dihydroethidium (DHE) fluorescence assay. Expression of 3-Nitrotyrosine (3-NT) was measured by western blot analysis. XBP1 knockdown was performed by Small RNA interference (siRNA). The knockdown efficiency was monitored by determining the protein level of XBP1 using western blot analysis. HUVECs were transfected with scramble siRNA or XBP1 siRNA using Lipofectamine 2000 for $6 \mathrm{~h}$, then treated with Nrf2 inducer tert-butylhydroquinone (tBHQ) $(20 \mu \mathrm{M})$ for $12 \mathrm{~h}$. The expressions of Nrf2 were determined by western blot analysis.

Results Overexpression of XBP-1 inhibited TNF- $\alpha$ induced ROS generation and 3-NT expression. Moreover, overexpression of XBP-1 enhanced $\mathrm{tBHO}$ induced Nrf2 expression. In addition, downregulation of XBP1 by transfection with XBP1 siRNA suppressed the tBHQ induced Nrf2 expression in cultured HUVECs.

Conclusions XBP1 suppresses TNF- $\alpha$ induced ROS generation possibly by activating Nrf2 in cultured HUVECs.

\section{GW23-e2215 ACTIVATION OF NF-E2-RELATED FACTOR 2 BY X-BOX BINDING PROTEIN 1 PROTECTS ENDOTHELIAL CELLS FROM TUMOUR NECROSIS FACTOR- $\alpha$ INDUCED OXIDATIVE STRESS}

doi:10.1136/heartjnl-2012-302920a.246

\footnotetext{
${ }^{1}$ Min Wang, ${ }^{1}$ Xiaoxian Qian, ${ }^{2}$ Sarah X Zhang, ${ }^{2}$ Josh J Wang. ${ }^{1}$ Department of Cardiology, The Third Affiliated Hospital, Sun Yat-sen University, Guangzhou, 510630, China; ${ }^{2}$ Harold Hamm Diabetes Center, University of Oklahoma Health Sciences Center, 941 Stanton L. Young Blvd., Oklahoma City, OK 73104, USA
}

Objectives Oxidative stress plays a critical role in endothelial cell (EC) dysfunction in atherosclerosis. NF-E2-related factor 2 (Nrf2) is the transcription factor which has antioxidant capacity. X-box binding protein 1 (XBP1) plays a great part in endoplasmic reticulum (ER) stress and ER stress response. This study is to test the hypothesis that spliced XBP1 has protective effect on tumour necrosis factor $\alpha$ (TNF- $\alpha$ ) induced oxidative stress, and the potential role of the activation of Nrf2 in cultured human umbilical vein endothelial cells (HUVECs).

Methods Spliced XBP1 was overexpressed in HUVECs by infection of cells with adenovirus encoding mouse spliced XBP1 (Ad-XBP1s). Adenovirus encoding green fluorescent protein (Ad-GFP) was used 